\title{
BMC Plant Biology reviewer acknowledgement 2014
}

\section{Catherine J Potenski}

Tabare Abadie

USA

Steffen Abel
Germany

Amanda Able

Australia

Gaber Abogadallah

Egypt

Maricelis Acevedo

USA

Anne-Françoise Adam-Blondon

France

Keith Adams

Canada

Oussama Ahrazem
Spain

Riccardo Aiese Cigliano

Spain

Karen Aitken

Australia

Takashi Akagi

Japan

Eduard Akhunov

USA

Magdy Alabady

USA

Salim Al-Babili

Saudi Arabia

Maria Albani

Germany
Nick Albert

New Zealand

Ruben Alcazar

Spain

Juan De Dios Alché

Spain

Ross Alexander

UK

Pablo Aleza

Spain

Francisco Alfocea

Spain

Andrew Allan

New Zealand

Doug Allen

USA

Jose M Alvarez

Spain

Sara Amancio

Portugal

Erik Andreasson

Sweden

Fernando Andres Lalaguna

Germany

Aldwin Anterola

USA

Carla Antonio

Portugal

Baltazar Antonio

Japan

\author{
Michael N Antoniou \\ UK \\ Pedro M Aparicio-Tejo \\ Spain \\ Rudi Appels \\ Australia \\ Miguel Aranda \\ Spain
}

Gen-Ichiro Arimura

Japan

Grace Armijo

Chile

Vincent Arondel

France

Michael Axtell

USA

Belay Ayele

Canada

Ricardo Azevedo

Brazil

Andreas Bachmair

Austria

Sacha Baginsky

Germany

Agnieszka Bagniewska-Zadworna Poland

Yuling Bai

Netherlands

Donovan Bailey

USA 
Fabienne Baillieul

France

Barbara Baker

USA

Salma Balazadeh

Germany

Alma Balestrazzi

Italy

Peter Balint-Kurti

USA

Tomohiro Ban

Japan

Pradeepa Bandaranayake

Sri Lanka

Zsófia Bánfalvi

Hungary

Abdelali Barakat

USA

Elena Baraldi

Italy

William Barbazuk

USA

Todd Barkman

USA

Yves Barriere

France

Francisco Barro

Spain

Daniele Bassi

Italy

Soumalee Basu

India

Giorgia Batelli

Italy

Sébastien Baud

France

Dirk Becker

Germany

Eric Beers

USA

Angjelina Belaj

Spain

Catherine Bellini

Sweden
Francois Belzile

Canada

Abdelhafid Bendahmane

France

Gary Bending

UK

Vagner Benedito

USA

Moussa Benhamed

France

Eva Benkova

Austria

Jeffrey Bennetzen

USA

Leonie Bentsink

Netherlands

Mark Bernards

Canada

Frank Bernhard

Germany

Alexandre Berr

France

James Berry

USA

Sébastien Besseau

France

K.E. Bett

Canada

Michael Bevan

UK

Brad Binder

USA

Benjamin Blonder

USA

Christine Boettcher

Australia

Martin Bohn

USA

Torsten Bohn

Luxembourg

Cordelia Bolle

Germany

Victoria Bonnecarrere

Uruguay
Guusje Bonnema

Netherlands

Ljudmilla Borisjuk

Germany

Maria Borisova-Mubarakshina

Russian Federation

Paul Boss

Australia

Rebecca Boston

USA

Javier Botto

Argentina

Alessandro Botton

Italy

Marc Boutry

Belgium

Peter Bozhkov

Sweden

Peter Bradbury

USA

Andrea Braeutigam

Germany

Gintaras Brazauskas

Lithuania

Phil Bregitzer

USA

Dev Britto

Canada

Mikael Brosche

Finland

Cory Brouwer

USA

Robert Brueggeman

USA

Qingyun Bu

China

Thomas Buckhout

Germany

Mariano Bulos

Argentina

Nicola Busatto

Italy

Daniel Bush

USA 
John Bussell

Australia

Patrick Byrne

USA

Francisco Cabrera-Chavez

Mexico

Tao Cai

China

Yingfan Cai

China

Myriam Calonje

Spain

Benjamin Campbell

USA

Michael Campbell

USA

Alexandre Campos

Portugal

Francisco R Canton

Spain

Dario Cantu

USA

Juan Carbonell

Spain

Pablo Carbonell-Bejerano

Spain

Maura Cardarelli

Italy

John Carman

USA

Sebastien Carpentier

Belgium

Joyce Cartagena

Japan

Clay Carter

USA

Carla Caruso

Italy

Luiz Carvalho

Brazil

Helena Carvalho

Portugal

Jorge Casal

Argentina
Simone Castellarin

Canada

David Chagne

New Zealand

Zhulong Chan

China

Yee-Yung Charng

Taiwan

Carolina Chavarro

USA

Kun-Song Chen

China

Shuangchen Chen

China

Dong-Hong Chen

China

Fadi Chen

China

Caiyan Chen

China

Fan Chen

China

Feng Chen

USA

Jinfeng Chen

USA

Michelle Y.Q. Chen

China

Yinglong Chen

China

Zhixiang Chen

USA

Riyan Cheng

USA

Ravindra Chibbar

Canada

Kevin Childs

USA

Maurizio Chiurazzi

Italy

Hyung-Taeg Cho

Korea, South

Giltsu Choi

Korea, South
Frederic Choulet

France

Wah Soon Chow

Australia

David Christopher

USA

Byung Yeoup Chung

Korea, South

Marta Cifuentes

Spain

Stephan Clemens

Germany

Sylvie Cloutier

Canada

Jeremy Coate

USA

Joseph Colasanti

Canada

David Collings

New Zealand

Thomas Colquhoun

USA

Eleonora Cominelli

Italy

Peter Constabel

Canada

Lucio Conti

Italy

Bret Cooper

USA

Sylvain Cordelier

France

Amandine Cornille

Sweden

Fabrizio Costa

Italy

Grant Cramer

USA

Martin Crespi

France

Xiangqin Cui

USA

Shaun Curtin

USA 


\begin{tabular}{|c|c|c|}
\hline $\begin{array}{l}\text { Abdelfattah Dababat } \\
\text { Turkey }\end{array}$ & $\begin{array}{l}\text { Laurent Deluc } \\
\text { USA }\end{array}$ & $\begin{array}{l}\text { Zhenying Dong } \\
\text { China }\end{array}$ \\
\hline $\begin{array}{l}\text { Hongyi Dai } \\
\text { China }\end{array}$ & $\begin{array}{l}\text { Xiuxin Deng } \\
\text { China }\end{array}$ & $\begin{array}{l}\text { Daolong Dou } \\
\text { China }\end{array}$ \\
\hline $\begin{array}{l}\text { Xinbin Dai } \\
\text { USA }\end{array}$ & $\begin{array}{l}\text { Emanuel Devers } \\
\text { Switzerland }\end{array}$ & $\begin{array}{l}\text { Renato D'Ovidio } \\
\text { Italy }\end{array}$ \\
\hline $\begin{array}{l}\text { Zhanwu Dai } \\
\text { France }\end{array}$ & $\begin{array}{l}\text { Paul Devlin } \\
\text { UK }\end{array}$ & $\begin{array}{l}\text { Max Dow } \\
\text { Ireland }\end{array}$ \\
\hline $\begin{array}{l}\text { Jonas Danielson } \\
\text { Sweden }\end{array}$ & $\begin{array}{l}\text { Michael K Deyholos } \\
\text { Canada }\end{array}$ & $\begin{array}{l}\text { Kurt Drickamer } \\
\text { UK }\end{array}$ \\
\hline $\begin{array}{l}\text { Zsuzsanna Darula } \\
\text { Hungary }\end{array}$ & $\begin{array}{l}\text { Christelle Deytieux } \\
\text { Belleau Reunion }\end{array}$ & $\begin{array}{l}\text { Hajk-Georg Drost } \\
\text { Germany }\end{array}$ \\
\hline $\begin{array}{l}\text { Sampa Das } \\
\text { India }\end{array}$ & $\begin{array}{l}\text { Sandrine Dhondt-Cordelier } \\
\text { France }\end{array}$ & $\begin{array}{l}\text { Arnaud Duhoux } \\
\text { France }\end{array}$ \\
\hline $\begin{array}{l}\text { Yasuhiro Date } \\
\text { Japan }\end{array}$ & $\begin{array}{l}\text { Christophe D'Hulst } \\
\text { France }\end{array}$ & $\begin{array}{l}\text { Marcello Duranti } \\
\text { Japan }\end{array}$ \\
\hline $\begin{array}{l}\text { Karine David } \\
\text { New Zealand }\end{array}$ & $\begin{array}{l}\text { Gabriele Di Gaspero } \\
\text { Italy }\end{array}$ & $\begin{array}{l}\text { Melvin Duvall } \\
\text { USA }\end{array}$ \\
\hline $\begin{array}{l}\text { Christopher Davies } \\
\text { Australia }\end{array}$ & $\begin{array}{l}\text { Daniel Dias } \\
\text { Australia }\end{array}$ & $\begin{array}{l}\text { Charles Dwamena } \\
\text { New Zealand }\end{array}$ \\
\hline $\begin{array}{l}\text { Kevin Davies } \\
\text { New Zealand }\end{array}$ & $\begin{array}{l}\text { Isabel Diaz } \\
\text { Spain }\end{array}$ & $\begin{array}{l}\text { Alan Eggenberger } \\
\text { USA }\end{array}$ \\
\hline $\begin{array}{l}\text { Stefan De Folter } \\
\text { Mexico }\end{array}$ & $\begin{array}{l}\text { Jose Diaz } \\
\text { Spain }\end{array}$ & $\begin{array}{l}\text { Malin Elfstrand } \\
\text { Sweden }\end{array}$ \\
\hline $\begin{array}{l}\text { Paolo De Franceschi } \\
\text { Italy }\end{array}$ & $\begin{array}{l}\text { Scott Diehn } \\
\text { USA }\end{array}$ & $\begin{array}{l}\text { Paula Elomaa } \\
\text { Finland }\end{array}$ \\
\hline $\begin{array}{l}\text { Luit J. De Kok } \\
\text { Netherlands }\end{array}$ & $\begin{array}{l}\text { Stephen Difazio } \\
\text { USA }\end{array}$ & $\begin{array}{l}\text { Francesco Emanuelli } \\
\text { Italy }\end{array}$ \\
\hline $\begin{array}{l}\text { Ruud De Maagd } \\
\text { Netherlands }\end{array}$ & $\begin{array}{l}\text { Yi Ding } \\
\text { China }\end{array}$ & $\begin{array}{l}\text { Eugenia Enfissi } \\
\text { UK }\end{array}$ \\
\hline $\begin{array}{l}\text { Nico De Storme } \\
\text { Belgium }\end{array}$ & $\begin{array}{l}\text { Assaf Distelfeld } \\
\text { Israel }\end{array}$ & $\begin{array}{l}\text { Jurgen Engelberth } \\
\text { USA }\end{array}$ \\
\hline $\begin{array}{l}\text { David De Vleesschauwer } \\
\text { Belgium }\end{array}$ & $\begin{array}{l}\text { Anna Dobritsa } \\
\text { USA }\end{array}$ & $\begin{array}{l}\text { Cawas Engineer } \\
\text { USA }\end{array}$ \\
\hline $\begin{array}{l}\text { Sacco De Vries } \\
\text { Netherlands }\end{array}$ & $\begin{array}{l}\text { Agnes Doligez } \\
\text { France }\end{array}$ & $\begin{array}{l}\text { Carmen Espinoza } \\
\text { Chile }\end{array}$ \\
\hline $\begin{array}{l}\text { Seth Debolt } \\
\text { USA }\end{array}$ & $\begin{array}{l}\text { Kara Dolinski } \\
\text { USA }\end{array}$ & $\begin{array}{l}\text { Yoram Eyal } \\
\text { Israel }\end{array}$ \\
\hline $\begin{array}{l}\text { Nicolas Delhomme } \\
\text { Sweden }\end{array}$ & $\begin{array}{l}\text { James Dombrowski } \\
\text { USA }\end{array}$ & $\begin{array}{l}\text { Muhammad Faheem } \\
\text { Pakistan }\end{array}$ \\
\hline $\begin{array}{l}\text { Costas Delis } \\
\text { Greece }\end{array}$ & $\begin{array}{l}\text { Lloyd Donaldson } \\
\text { New Zealand }\end{array}$ & $\begin{array}{l}\text { Osvaldo Failla } \\
\text { Italy }\end{array}$ \\
\hline $\begin{array}{l}\text { Serge Delrot } \\
\text { France }\end{array}$ & $\begin{array}{l}\text { Aiwu Dong } \\
\text { China }\end{array}$ & $\begin{array}{l}\text { Aaron Fait } \\
\text { Israel }\end{array}$ \\
\hline
\end{tabular}


Chengming Fan
China

Liumin Fan

China

David Fang

USA

Eva Farre

USA

Jacqueline Farrell

USA

Attila Fehér

Hungary

Alejandro Ferrando

Spain

Alison Ferrie

Canada

Jan Fíla

Czech Republic

Sophie Filleur

France

Richard Finkers

Netherlands

Matthias Fladung

Germany

Andrew Flavell

UK

Jozsef Fodor

Hungary

Daniel Fonceka

France

Christopher Ford

Australia

Britta Forster

Australia

John Forster

Australia

Christine Foyer

UK

Michael Francki

Australia

Jerome Franckowiak

USA

Wolfgang Frank

Germany
Noni Franklin-Tong

UK

Susanne Franssen

Germany

Elisabetta Frascaroli

Italy

Margrit Frentzen

Germany

Florian Frugier

France

Julia Frugoli

USA

Daolin Fu

China

Feng-Ling Fu

China

Binying Fu

China

Yong-Fu Fu

China

Karen Fugate

USA

Hiroaki Fujii

Finland

Yasunari Fujita

Japan

Hiroyuki Fukuoka

Japan

Beata Gabrys

Poland

Agata Gadaleta

Italy

Giovanni Gadda

USA

Malgorzata Gaj

Poland

Carlos Galeano

Belgium

Gabor Galiba

Hungary

Ivan Galis

Japan

Giulio Galla

Italy
Vanessa Galli

Brazil

Susheng Gan

USA

Dongying Gao

USA

Junping Gao

China

Liangliang Gao

USA

Nigel Gapper

USA

Rohini Garg

India

Nicole Gaude

Germany

Danny Geelen

Belgium

Agustina Gentile

Brazil

Bernard Genty

France

Godelieve Gheysen

Belgium

Luca Gianfranceschi

Italy

Antonia Gibalova

Czech Republic

Markus Gierth

Germany

Javier Gil-Humanes

USA

Upinder Gill

USA

Lara Giongo

Italy

Giovanni Giuliano

Italy

Jeff Glaubitz

USA

Fred Gmitter

USA

Barry Goldfarb

USA 
Vassiliy Goltsev

Bulgaria

John Golz

Australia

Nikolay P. Goncharov

Russian Federation

Daniel H. Gonzalez

Argentina

Carmen González

Spain

Luis González-Candelas

Spain

William Gordon-Kamm

USA

Francoise Gosti

France

Lydia Gramzow

Germany

Silvana Grandillo

Italy

Antonio Granell

Spain

David Grant

USA

Eric Grenier

France

Peter Gresshoff

Australia

Åsa Grimberg

Sweden

Andrew Groover

USA

Erich Grotewold

USA

Corrinne Grover

USA

Nicole Gruenheit

UK

Yong Qiang Gu

USA

Paul Gugger

USA

Sabine Guillaumie

France
Raghavendra Gunnaiah

India

Yan Guo

China

Hui Guo

USA

Om Gupta

India

Pushpendra Gupta

India

Juan Gutierrez-Gonzalez

USA

Jose Gutierrez-Marcos

UK

Carlos Guzman

Spain

Niclas Gyllenstrand

Sweden

János Györgyey

Hungary

Jeff Habben

USA

Charles Hachez

Belgium

Bernd Hackauf

Germany

Uwe Hacke

Canada

Ken Haga

Japan

Hely Häggman

Finland

Candace Haigler

USA

Ian Hallett

New Zealand

Noureddine Hamamouch

USA

Bjoern Hamberger

Denmark

Mei Han

Canada

Oksoo Han

Korea, South
Yuepeng Han

China

Yuko Hanba

Japan

Robert Hancock

UK

Marc Hanikenne

Belgium

Viola Hanke

Germany

L. Curtis Hannah

USA

Abdelali Hannoufa

Canada

Maureen Hanson

USA

Yoshie Hanzawa

USA

Phillip Harries

USA

Neil Harris

Canada

Philip Harris

New Zealand

Caroline Hartmann

France

Marie-Theres Hauser

Austria

Simon Hawkins

France

Matthew Haworth

Italy

Samuel Hazen

USA

Guangyuan He

China

Ping He

USA

Yuehui He

China

Yuke He

China

Zhonghu He

China 
Reinhard Hehl

Germany

Alain Hehn

France

Martin Heil

Germany

Jan Hejatko

Czech Republic

Roger Hellens

Australia

Kian Hematy

France

Tracie Hennen-Bierwagen

USA

Jacek Hennig

Poland

Luis E. Hernandez

Spain

Georgina Hernández

Mexico

Sigrid Heuer

Australia

Tarek Hewezi

USA

Julian Hibberd

UK

Karen Hicks

USA

Oriane Hidalgo

UK

Éva Hideg

Hungary

Angelika Hilbeck

Switzerland

Henk Hilhorst

Netherlands

Ross Hill

Australia

Bradley Hillman

USA

Medrano Hipolito

Spain

Bertrand Hirel

France
Candice Hirsch

USA

Cory Hirsch

USA

Joseph Hirschberg

Israel

Ute Hoecker

Germany

Susanne Hoffmann-Benning

USA

Monica Hofte

Belgium

Annette Hohe

Germany

Yiguo Hong

China

Sharon Hook

Australia

Jim Horn

USA

Matthew Horton

Austria

David Horvath

USA

Li-Ching Hsieh

Taiwan

Jianping $\mathbf{H u}$

USA

Jinling Huang

USA

Zhenying Huang

China

Karen Hudson

USA

Alisa Huffaker

USA

Matthew Hufford

USA

Edgar Huitema

UK

Cristian Ibanez

Chile

Yoko Iijima

Japan
Tatsuya Ikeda

Japan

Richard Immink

Netherlands

Claudio Inostroza-Blancheteau

Chile

Rosy Isaias

Brazil

Nobuaki Ishida

Japan

Yasuhiro Ito

Japan

Norberto Iusem

Argentina

Rumen Ivanov

Germany

Hiroyoshi Iwata

Japan

Kunio Iwatsuki

Japan

Niranjani Iyer

USA

Takeshi Izawa

Japan

Jeanne Jacobs

New Zealand

Dan Jacobson

South Africa

Guru Jagadeeswaran

USA

Pradeep Jain

India

Malgorzata Jakubowicz

Poland

Georg Jander

USA

Robert Jansen

USA

Dennis Janz

Germany

Jose Jarillo

Spain

Paul Jarvis

UK 


\begin{tabular}{|c|c|c|}
\hline $\begin{array}{l}\text { Amir Reza Jassbi } \\
\text { Japan }\end{array}$ & $\begin{array}{l}\text { Lei Kai } \\
\text { Germany }\end{array}$ & $\begin{array}{l}\text { Felix Kessler } \\
\text { Switzerland }\end{array}$ \\
\hline $\begin{array}{l}\text { Eric Jenczewski } \\
\text { UK }\end{array}$ & $\begin{array}{l}\text { Vijaya Gopal Kakani } \\
\text { USA }\end{array}$ & $\begin{array}{l}\text { Zhang Kewei } \\
\text { China }\end{array}$ \\
\hline $\begin{array}{l}\text { Pablo Jenik } \\
\text { USA }\end{array}$ & $\begin{array}{l}\text { Peter Kalo } \\
\text { Hungary }\end{array}$ & $\begin{array}{l}\text { Awais Khan } \\
\text { Peru }\end{array}$ \\
\hline $\begin{array}{l}\text { Martin Jensen } \\
\text { Denmark }\end{array}$ & $\begin{array}{l}\text { Sekhar Kambakam } \\
\text { USA }\end{array}$ & $\begin{array}{l}\text { Aruna Kilaru } \\
\text { USA }\end{array}$ \\
\hline $\begin{array}{l}\text { Soon-Chun Jeong } \\
\text { Korea, South }\end{array}$ & $\begin{array}{l}\text { Radek Kana } \\
\text { Czech Republic }\end{array}$ & $\begin{array}{l}\text { Graham King } \\
\text { Australia }\end{array}$ \\
\hline $\begin{array}{l}\text { Holger Jeske } \\
\text { Germany }\end{array}$ & $\begin{array}{l}\text { Angelos Kanellis } \\
\text { Greece }\end{array}$ & $\begin{array}{l}\text { Edward Kirby } \\
\text { USA }\end{array}$ \\
\hline $\begin{array}{l}\text { Heyuan Jiang } \\
\text { China }\end{array}$ & $\begin{array}{l}\text { Byung-Ho Kang } \\
\text { USA }\end{array}$ & $\begin{array}{l}\text { Tatjana Kleine } \\
\text { Germany }\end{array}$ \\
\hline $\begin{array}{l}\text { Ning Jiang } \\
\text { USA }\end{array}$ & $\begin{array}{l}\text { Hunseung Kang } \\
\text { Korea, South }\end{array}$ & $\begin{array}{l}\text { Helmut Knüpffer } \\
\text { Germany }\end{array}$ \\
\hline $\begin{array}{l}\text { Xingyu Jiang } \\
\text { China }\end{array}$ & $\begin{array}{l}\text { Hong-Gu Kang } \\
\text { USA }\end{array}$ & $\begin{array}{l}\text { Takanori Kobayashi } \\
\text { Japan }\end{array}$ \\
\hline $\begin{array}{l}\text { Zhiqiang Jin } \\
\text { China }\end{array}$ & $\begin{array}{l}\text { Merijn Kant } \\
\text { Netherlands }\end{array}$ & $\begin{array}{l}\text { Koichi Kobayashi } \\
\text { Japan }\end{array}$ \\
\hline $\begin{array}{l}\text { Biao Jin } \\
\text { China }\end{array}$ & $\begin{array}{l}\text { Michael Kantar } \\
\text { USA }\end{array}$ & $\begin{array}{l}\text { Michael Kobor } \\
\text { Canada }\end{array}$ \\
\hline $\begin{array}{l}\text { Jian Jin } \\
\text { China }\end{array}$ & $\begin{array}{l}\text { Kostya Kanyuka } \\
\text { UK }\end{array}$ & $\begin{array}{l}\text { Andriy Kochevenko } \\
\text { Germany }\end{array}$ \\
\hline $\begin{array}{l}\text { Hai-Chun Jing } \\
\text { China }\end{array}$ & $\begin{array}{l}\text { Rick Karban } \\
\text { USA }\end{array}$ & $\begin{array}{l}\text { Gábor Kocsy } \\
\text { Hungary }\end{array}$ \\
\hline $\begin{array}{l}\text { Russell Jones } \\
\text { USA }\end{array}$ & $\begin{array}{l}\text { Surekha Katiyar-Agarwal } \\
\text { India }\end{array}$ & $\begin{array}{l}\text { Ajay Kohli } \\
\text { Philippines }\end{array}$ \\
\hline $\begin{array}{l}\text { Matthieu Henri Antoon Jozef Joosten } \\
\text { Netherlands }\end{array}$ & $\begin{array}{l}\text { Kerstin Kaufmann } \\
\text { Germany }\end{array}$ & $\begin{array}{l}\text { Bryan Kolaczkowski } \\
\text { USA }\end{array}$ \\
\hline $\begin{array}{l}\text { Angela Juhasz } \\
\text { Hungary }\end{array}$ & $\begin{array}{l}\text { Jagreet Kaur } \\
\text { India }\end{array}$ & $\begin{array}{l}\text { Frederic Kolb } \\
\text { USA }\end{array}$ \\
\hline $\begin{array}{l}\text { Je Hyeong Jung } \\
\text { USA }\end{array}$ & $\begin{array}{l}\text { Nat Kav } \\
\text { Canada }\end{array}$ & $\begin{array}{l}\text { David Kopecky } \\
\text { Czech Republic }\end{array}$ \\
\hline $\begin{array}{l}\text { Geunhwa Jung } \\
\text { USA }\end{array}$ & $\begin{array}{l}\text { Tsutomu Kawasaki } \\
\text { Japan }\end{array}$ & $\begin{array}{l}\text { Helena Korpelainen } \\
\text { Finland }\end{array}$ \\
\hline $\begin{array}{l}\text { Parijat Juvale } \\
\text { USA }\end{array}$ & $\begin{array}{l}\text { Kemal Kazan } \\
\text { Australia }\end{array}$ & $\begin{array}{l}\text { Jens Kossmann } \\
\text { South Africa }\end{array}$ \\
\hline $\begin{array}{l}\text { Piotr Kachlicki } \\
\text { Poland }\end{array}$ & $\begin{array}{l}\text { Julia Kehr } \\
\text { Spain }\end{array}$ & $\begin{array}{l}\text { Simeon Kotchoni } \\
\text { USA }\end{array}$ \\
\hline $\begin{array}{l}\text { Pradeep Kachroo } \\
\text { USA }\end{array}$ & $\begin{array}{l}\text { Julia Kehr } \\
\text { Germany }\end{array}$ & $\begin{array}{l}\text { Stefanos Koundouras } \\
\text { Greece }\end{array}$ \\
\hline $\begin{array}{l}\text { Joanna Kacprzyk } \\
\text { Ireland }\end{array}$ & $\begin{array}{l}\text { Beat Keller } \\
\text { Switzerland }\end{array}$ & $\begin{array}{l}\text { Kanako Koyanagi } \\
\text { Japan }\end{array}$ \\
\hline
\end{tabular}


Elena Kramer

USA

Gabi Krczal

Germany

\section{Sergey Krupenko}

USA

Karl Kugler

Germany

Masahiko Kumagai

Japan

V. Dinesh Kumar

India

Sanjay Kumar

India

Shigeichi Kumazaki

Japan

Jochen Kumlehn

Germany

Carlos Labate

Brazil

Maite Lacuesta

Spain

Zhongxiong Lai

China

Zhibing Lai

USA

Thierry Langin

France

Maria Valeria Lara

Argentina

Debbie Laudencia-Chingcuanco USA

Stefan Laurent

Germany

Virginie Lauvergeat

France

Thomas Laux

Germany

Erwan Le Deunff

France

Peter John Lea

UK

Tong Geon Lee

USA
Jong Kyu Lee

Korea, South

Suk-Ha Lee

Korea, South

Matti Leino

Sweden

Andrew Leitch

UK

Janne Lempe

Germany

Michael Lenhard

Germany

Bosheng Li

USA

Chenghao Li

China

Jiaru Li

China

Lei Li

USA

Huihui Li

China

Maoteng Li

China

Yuhua Li

China

Shutian Li

Germany

Song Li

USA

Hong-Ye Li

China

Ting Li

USA

Yan Li

China

Ying Li

USA

Yongfang Li

USA

Yongle Li

Australia

Guohua Liang

China
Marc Libault

USA

David Lightfoot

USA

Wilco Ligterink

Netherlands

Jun Lim

Korea, South

Pyung Ok Lim

Korea, South

Chentao Lin

USA

Jinxing Lin

China

Kui Lin

China

Yao-Cheng Lin

Belgium

Hong-Qing Ling

China

Alexander Lipka

USA

Jinjie Liu

USA

Jianquan Liu

China

Qingpo Liu

China

Shengyi Liu

China

Lai-Hua Liu

China

Wen-Zhe Liu

China

Renyi Liu

USA

Renyi Liu

China

Xigang Liu

China

Zhongchi Liu

USA

Alan Lloyd

USA 
Clive Lo

Hong Kong

Maria Logacheva

Russian Federation

Susan Lolle

Canada

Jose Lopez-Bucio

Mexico

Rodolfo Lopez-Gomez

Mexico

Ann Loraine

Aaron Lorenz

USA

Rafael Lozano

Spain

Hua Lu

USA

Yingmin Lu

China

Uwe Ludewig

Germany

Jutta Ludwig-Müller

Germany

Yan Luo

China

Qing-Hu Ma

China

Wujun Ma

Australia

Marco Maccaferri

Italy

Emma Mace

Australia

Milagros Machinandiarena

Argentina

David Mackey

USA

David Mackill

USA

Richard Colin Macknight

New Zealand

Takaki Maekawa

Germany
Massimo Maffei

Italy

Jurandir Magalhaes

Brazil

Ivan Maia

Brazil

Indu Maiti

USA

Mario Malagoli

Italy

Abul Kalam Mandal

India

Long Mao

China

Daunay Marie-Christine

France

Soma Marla

India

Cathie Martin

UK

Antonio Martín

Spain

Pedro Martinez-Gomez

Spain

José M Martinez-Zapater

Spain

Enrico Martinoia

Switzerland

Martin Mascher

Germany

Stefania Masci

Italy

Esten Mason

USA

Anna Maria Mastrangelo Italy

Ulrike Mathesius

Australia

Andrea Matros

Germany

Fumio Matsuda

Japan

Hideaki Matsumoto

Japan
Roberto Mattioli

Italy

Tim Mauchline

UK

Brigitte Mauch-Mani

Switzerland

Ivan Maureira Butler

Chile

Klaus Mayer

Germany

Christian Mazars

France

Michael Mazourek

USA

Martin Robert Mcainsh

UK

Bruce Mcclure

USA

Karen Mcginnis

USA

J. Mitchell Mcgrath

USA

Leah Mchale

USA

Michael Mcmullen

USA

Joel Mcneal

USA

Kevin Mcphee

USA

Andy Meharg

UK

David Meinke

USA

Charles Melnyk

UK

Rainer Melzer

Germany

David Mendoza

USA

Yijun Meng

China

Zheng Meng

China 
Marcelo Menossi

Brazil

Kristin Mercer

USA

Irute Meskiene

Austria

Jean-Pierre Metraux

Switzerland

Eli Meyer

USA

Rhonda Meyer

Germany

Blake Meyers

USA

Thomas Miedaner

Germany

Tony Millar

Australia

Tony Miller

UK

Joel Milner

UK

Ray Ming

USA

Mehdi Mirzaei

Australia

Richard Mithen

UK

Juliane Mittasch

Germany

Yutaka Miyazawa

Japan

Hans-Peter Mock

Germany

Wolfgang Moeder

Canada

Alejandra Moenne

Chile

Yvan Moënne-Loccoz

France

Volker Mohler

Germany

Ian Max Møller

Denmark
Tapan Mondal

India

Paula Maria Moolhuijzen

Australia

Richard Moore

USA

María De La Luz Mora

Chile

Raphael Morillon

Spain

Panagiotis Moschou

Sweden

Reiko Motohashi

Japan

Zhonglin Mou

USA

Katriina Mouhu

Finland

Iva Mozgova

Sweden

Wellington Muchero

USA

Gary Muehlbauer

USA

Douglas Muench

Canada

Sunil Mukherjee

India

Philip Mullineaux

UK

Sujatha Mulpuri

India

Stéphane Muños

France

Luis Mur

UK

Koji Murai

Japan

Koji Muramoto

Japan

Alex Murphy

UK

Denis Murphy

UK
Serge Muyldermans

Belgium

Sean Myles

Canada

Rachel Naegele

USA

Istvan Nagy

Hungary

Ken Naito

Japan

Kenta Nakai

Japan

Norihito Nakamichi

Japan

Kazuo Nakashima

Japan

Eiji Nambara

Canada

Simona Nardozza

New Zealand

Javier Narvaez-Vasquez

USA

Utpal Nath

India

Alireza Navabi

Canada

Suresh Neethirajan

Canada

Karlie Neilson

Australia

David Nelson

USA

Lev Nemchinov

USA

Madhav Nepal

USA

Carl Ng

Ireland

Henry Nguyen

USA

Dahlia Nielsen

USA

Tom Hamborg Nielsen

Denmark 
Manuel Nieves-Cordones

France

Tomotaro Nishikawa

Japan

Takumi Nishiuchi

Japan

Ill-Sup Nou

Korea, South

Thomas Nussbaumer

Austria

Sergio J Ochatt

France

Francis Ogbonnaya

Australia

John Ohlrogge

USA

Akemi Ohmiya

Japan

Takashi Okada

Australia

Elina Oksanen

Finland

Kazutoshi Okuno

Japan

Richard Olmstead

USA

Matthew Olson

USA

Satoshi Oota

Japan

David Oppenheimer

USA

Bernardo Ordas

Spain

Jamie O'Rourke

USA

Cristina Ortega-Villasante

Spain

Anne Osbourn

UK

Eileen O'Toole

USA

Primoz Oven

Slovenia
Hakan Ozkan

Turkey

Natalia Pabon Mora

Colombia

Jordon Pace

USA

Chiara Pagliarani

Italy

Magda Pál

Hungary

Norman Pammenter

Suriname

Jianwei Pan

China

Sona Pandey

USA

Ralph Panstruga

Germany

Spiros Papakostas

Finland

Roger Parish

Australia

Gisele Passaia

Brazil

Maria Marta Pastina

Brazil

Victoria Pastor

Switzerland

Matthew Paul

UK

Katharina Pawlowski

Sweden

Tomasz Pawlowski

Poland

Wojtek Pawlowski

USA

Raja Payyavula

USA

Mario Enrico Pè

Italy

Scott Peck

USA

Xinwu Pei

China
Suprasanna Penna

India

Deepak Pental

India

Andy Pereira

USA

Silvia Pereyra

Uruguay

Begona Perez-Vich

Sudan

Serena Perilli

Netherlands

Dragan Perovic

Germany

Christoph Peterhansel

Germany

Sander Peters

Netherlands

Thomas Peterson

USA

Mario Pezzotti

Italy

Anh Pham

USA

Romain Philippe

France

Pietro Piffanelli

Italy

Sara Pinosio

Italy

J. Chris Pires

USA

Frederic Pitre

Canada

Georg Pohnert

Germany

Benoit Poinssot

France

Danijela Poljuha

Croatia

Andrea Polle

Germany

Mikhail M. Pooggin

Switzerland 


\begin{tabular}{|c|c|c|}
\hline $\begin{array}{l}\text { Zoe Popper } \\
\text { Ireland }\end{array}$ & $\begin{array}{l}\text { R. George Ratcliffe } \\
\text { UK }\end{array}$ & $\begin{array}{l}\text { William Rooney } \\
\text { USA }\end{array}$ \\
\hline $\begin{array}{l}\text { David Pot } \\
\text { USA }\end{array}$ & $\begin{array}{l}\text { Milind Ratnaparkhe } \\
\text { India }\end{array}$ & $\begin{array}{l}\text { Luis Mauro Rosa } \\
\text { Brazil }\end{array}$ \\
\hline $\begin{array}{l}\text { Sreedhara Ashok Prabhu } \\
\text { India }\end{array}$ & $\begin{array}{l}\text { Catherine Ravel } \\
\text { France }\end{array}$ & $\begin{array}{l}\text { Jocelyn Rose } \\
\text { USA }\end{array}$ \\
\hline $\begin{array}{l}\text { Manoj Prasad } \\
\text { India }\end{array}$ & $\begin{array}{l}\text { Bianca Reeksting } \\
\text { South Africa }\end{array}$ & $\begin{array}{l}\text { Ray J. Rose } \\
\text { Australia }\end{array}$ \\
\hline $\begin{array}{l}\text { Jill Preston } \\
\text { USA }\end{array}$ & $\begin{array}{l}\text { Tony Remans } \\
\text { Belgium }\end{array}$ & $\begin{array}{l}\text { Rebecca Roston } \\
\text { USA }\end{array}$ \\
\hline $\begin{array}{l}\text { Holger Puchta } \\
\text { Germany }\end{array}$ & $\begin{array}{l}\text { Maria Remis } \\
\text { Argentina }\end{array}$ & $\begin{array}{l}\text { Mathieu Rouard } \\
\text { France }\end{array}$ \\
\hline $\begin{array}{l}\text { Stanislawa Pukacka } \\
\text { Poland }\end{array}$ & $\begin{array}{l}\text { Hugues Renault } \\
\text { France }\end{array}$ & $\begin{array}{l}\text { Steve Rounsley } \\
\text { USA }\end{array}$ \\
\hline $\begin{array}{l}\text { Jo Putterill } \\
\text { New Zealand }\end{array}$ & $\begin{array}{l}\text { Ralf Reski } \\
\text { Germany }\end{array}$ & $\begin{array}{l}\text { Matthew Rouse } \\
\text { USA }\end{array}$ \\
\hline $\begin{array}{l}\text { Xiaoquan Qi } \\
\text { China }\end{array}$ & $\begin{array}{l}\text { Manuel Rey } \\
\text { Spain }\end{array}$ & $\begin{array}{l}\text { Heather Rowe } \\
\text { Canada }\end{array}$ \\
\hline $\begin{array}{l}\text { Xiaoquan Qi } \\
\text { UK }\end{array}$ & $\begin{array}{l}\text { Jose Luis Reyes } \\
\text { Mexico }\end{array}$ & $\begin{array}{l}\text { Swarup Roy Choudhury } \\
\text { USA }\end{array}$ \\
\hline $\begin{array}{l}\text { Feng Qin } \\
\text { China }\end{array}$ & $\begin{array}{l}\text { Arantza Rico } \\
\text { UK }\end{array}$ & $\begin{array}{l}\text { Wilfried Rozhon } \\
\text { Austria }\end{array}$ \\
\hline $\begin{array}{l}\text { Wenping Qiu } \\
\text { USA }\end{array}$ & $\begin{array}{l}\text { Christoph Ringli } \\
\text { Switzerland }\end{array}$ & $\begin{array}{l}\text { Markus Ruhsam } \\
\text { UK }\end{array}$ \\
\hline $\begin{array}{l}\text { Le Qing Qu } \\
\text { China }\end{array}$ & $\begin{array}{l}\text { Rosa M Rivero } \\
\text { Spain }\end{array}$ & $\begin{array}{l}\text { Daniel Runcie } \\
\text { USA }\end{array}$ \\
\hline $\begin{array}{l}\text { Ronaldo Quaggio } \\
\text { Brazil }\end{array}$ & $\begin{array}{l}\text { Dae-Kyun Ro } \\
\text { Canada }\end{array}$ & $\begin{array}{l}\text { John Runions } \\
\text { UK }\end{array}$ \\
\hline $\begin{array}{l}\text { Silvia Quaggiotti } \\
\text { Italy }\end{array}$ & $\begin{array}{l}\text { Helene Robert } \\
\text { Czech Republic }\end{array}$ & $\begin{array}{l}\text { Mark Running } \\
\text { USA }\end{array}$ \\
\hline $\begin{array}{l}\text { Marie-Christine Quillet } \\
\text { France }\end{array}$ & $\begin{array}{l}\text { Irma Roberts } \\
\text { Argentina }\end{array}$ & $\begin{array}{l}\text { Bob Rutledge } \\
\text { Canada }\end{array}$ \\
\hline $\begin{array}{l}\text { Sathishkumar Ramalingam } \\
\text { India }\end{array}$ & $\begin{array}{l}\text { Monica Rodriguez } \\
\text { Italy }\end{array}$ & $\begin{array}{l}\text { Peter Ryan } \\
\text { Australia }\end{array}$ \\
\hline $\begin{array}{l}\text { Kanniah Rajasekaran } \\
\text { USA }\end{array}$ & $\begin{array}{l}\text { Carlos Marcelino Rodriguez Lopez } \\
\text { Australia }\end{array}$ & $\begin{array}{l}\text { Francois Sabot } \\
\text { France }\end{array}$ \\
\hline $\begin{array}{l}\text { Randeep Rakwal } \\
\text { Japan }\end{array}$ & $\begin{array}{l}\text { Adrienne Roeder } \\
\text { USA }\end{array}$ & $\begin{array}{l}\text { Melanie Sacco } \\
\text { USA }\end{array}$ \\
\hline $\begin{array}{l}\text { Harsh Raman } \\
\text { Australia }\end{array}$ & $\begin{array}{l}\text { William John Rogers } \\
\text { Argentina }\end{array}$ & $\begin{array}{l}\text { Gavin Sacks } \\
\text { USA }\end{array}$ \\
\hline $\begin{array}{l}\text { Jean-Francois Rami } \\
\text { France }\end{array}$ & $\begin{array}{l}\text { Charles Romieu } \\
\text { France }\end{array}$ & $\begin{array}{l}\text { Ari Sadanandom } \\
\text { UK }\end{array}$ \\
\hline $\begin{array}{l}\text { Xie Rangjin } \\
\text { China }\end{array}$ & $\begin{array}{l}\text { Ann Christin Rönnberg-Wästljung } \\
\text { Sweden }\end{array}$ & $\begin{array}{l}\text { Kari Saikkonen } \\
\text { Finland }\end{array}$ \\
\hline
\end{tabular}




\begin{tabular}{|c|c|c|}
\hline $\begin{array}{l}\text { Katsumi Sakata } \\
\text { Japan }\end{array}$ & $\begin{array}{l}\text { Gerald Schoenknecht } \\
\text { USA }\end{array}$ & $\begin{array}{l}\text { Libo Shan } \\
\text { USA }\end{array}$ \\
\hline $\begin{array}{l}\text { Keyan Salzman } \\
\text { USA }\end{array}$ & $\begin{array}{l}\text { Monika Schreiner } \\
\text { Germany }\end{array}$ & $\begin{array}{l}\text { Eilon Shani } \\
\text { Israel }\end{array}$ \\
\hline $\begin{array}{l}\text { Deborah Samac } \\
\text { USA }\end{array}$ & $\begin{array}{l}\text { Gunda Schulte Auf'M Erley } \\
\text { Germany }\end{array}$ & $\begin{array}{l}\text { Thomas Sharkey } \\
\text { USA }\end{array}$ \\
\hline $\begin{array}{l}\text { Jozef Samaj } \\
\text { Czech Republic }\end{array}$ & $\begin{array}{l}\text { Wilfried Schwab } \\
\text { Germany }\end{array}$ & $\begin{array}{l}\text { Peter Sharp } \\
\text { Australia }\end{array}$ \\
\hline $\begin{array}{l}\text { Robert Sammons } \\
\text { USA }\end{array}$ & $\begin{array}{l}\text { Jorg Schwender } \\
\text { USA }\end{array}$ & $\begin{array}{l}\text { Yuri Shavrukov } \\
\text { Australia }\end{array}$ \\
\hline $\begin{array}{l}\text { Marcus Samuel } \\
\text { Canada }\end{array}$ & $\begin{array}{l}\text { Steven Scofield } \\
\text { USA }\end{array}$ & $\begin{array}{l}\text { Cheng Shihua } \\
\text { China }\end{array}$ \\
\hline $\begin{array}{l}\text { Gerardo Sánchez } \\
\text { Argentina }\end{array}$ & $\begin{array}{l}\text { Marek Sebela } \\
\text { Czech Republic }\end{array}$ & $\begin{array}{l}\text { Toshiharu Shikanai } \\
\text { Japan }\end{array}$ \\
\hline $\begin{array}{l}\text { Paloma Sanchez-Bel } \\
\text { Spain }\end{array}$ & $\begin{array}{l}\text { Ronald Sederoff } \\
\text { USA }\end{array}$ & $\begin{array}{l}\text { Kaori Shiojiri } \\
\text { Japan }\end{array}$ \\
\hline $\begin{array}{l}\text { Devinder Sandhu } \\
\text { USA }\end{array}$ & $\begin{array}{l}\text { Armand Seguin } \\
\text { Canada }\end{array}$ & $\begin{array}{l}\text { Katsuhiro Shiratake } \\
\text { Japan }\end{array}$ \\
\hline $\begin{array}{l}\text { Mirella Sari Gorla } \\
\text { Italy }\end{array}$ & $\begin{array}{l}\text { Verena Seidl-Seiboth } \\
\text { Austria }\end{array}$ & $\begin{array}{l}\text { Qing Yan Shu } \\
\text { China }\end{array}$ \\
\hline $\begin{array}{l}\text { Tomasz Sarnowski } \\
\text { Poland }\end{array}$ & $\begin{array}{l}\text { Georg Seifert } \\
\text { Austria }\end{array}$ & $\begin{array}{l}\text { Bin Shuai } \\
\text { USA }\end{array}$ \\
\hline $\begin{array}{l}\text { Hidenori Sassa } \\
\text { Japan }\end{array}$ & $\begin{array}{l}\text { Yasuyo Sekiyama } \\
\text { Japan }\end{array}$ & $\begin{array}{l}\text { Jeffry Shultz } \\
\text { USA }\end{array}$ \\
\hline $\begin{array}{l}\text { Kazuhiro Sato } \\
\text { Japan }\end{array}$ & $\begin{array}{l}\text { David Selinger } \\
\text { USA }\end{array}$ & $\begin{array}{l}\text { Cao Shuqing } \\
\text { China }\end{array}$ \\
\hline $\begin{array}{l}\text { Arnould Savoure } \\
\text { France }\end{array}$ & $\begin{array}{l}\text { Jennifer Selinski } \\
\text { Germany }\end{array}$ & $\begin{array}{l}\text { Richard Sicher } \\
\text { USA }\end{array}$ \\
\hline $\begin{array}{l}\text { Samir Sawant } \\
\text { India }\end{array}$ & $\begin{array}{l}\text { Marc-André Selosse } \\
\text { France }\end{array}$ & $\begin{array}{l}\text { Imran Siddiqi } \\
\text { India }\end{array}$ \\
\hline $\begin{array}{l}\text { Rachit Saxena } \\
\text { India }\end{array}$ & $\begin{array}{l}\text { Champa Sengupta-Gopalan } \\
\text { USA }\end{array}$ & $\begin{array}{l}\text { Lyudmila Sidorenko } \\
\text { UK }\end{array}$ \\
\hline $\begin{array}{l}\text { Henk Schat } \\
\text { Netherlands }\end{array}$ & $\begin{array}{l}\text { Muthappa Senthil-Kumar } \\
\text { India }\end{array}$ & $\begin{array}{l}\text { Daniela Sieh } \\
\text { Germany }\end{array}$ \\
\hline $\begin{array}{l}\text { Günther Scherer } \\
\text { Germany }\end{array}$ & $\begin{array}{l}\text { Shigemi Seo } \\
\text { Japan }\end{array}$ & $\begin{array}{l}\text { Herman Silva } \\
\text { Chile }\end{array}$ \\
\hline $\begin{array}{l}\text { John Schiefelbein } \\
\text { USA }\end{array}$ & $\begin{array}{l}\text { Kjell Sergeant } \\
\text { Luxembourg }\end{array}$ & $\begin{array}{l}\text { Thierry Simonneau } \\
\text { France }\end{array}$ \\
\hline $\begin{array}{l}\text { Florian P Schiestl } \\
\text { Switzerland }\end{array}$ & $\begin{array}{l}\text { Andrew Severin } \\
\text { USA }\end{array}$ & $\begin{array}{l}\text { Anil Singh } \\
\text { India }\end{array}$ \\
\hline $\begin{array}{l}\text { Jos Schippers } \\
\text { Germany }\end{array}$ & $\begin{array}{l}\text { Jyoti Shah } \\
\text { USA }\end{array}$ & $\begin{array}{l}\text { Dharmendra Singh } \\
\text { USA }\end{array}$ \\
\hline $\begin{array}{l}\text { Philipp Schlüter } \\
\text { Switzerland }\end{array}$ & $\begin{array}{l}\text { Zaigham Shahzad } \\
\text { France }\end{array}$ & $\begin{array}{l}\text { Mohan Singh } \\
\text { Australia }\end{array}$ \\
\hline
\end{tabular}




\begin{tabular}{|c|c|c|}
\hline $\begin{array}{l}\text { Alok Krishna Sinha } \\
\text { India }\end{array}$ & $\begin{array}{l}\text { C Neal Stewart } \\
\text { USA }\end{array}$ & $\begin{array}{l}\text { Yoshikazu Tanaka } \\
\text { Japan }\end{array}$ \\
\hline $\begin{array}{l}\text { Aleksandra Skirycz } \\
\text { Brazil }\end{array}$ & $\begin{array}{l}\text { Annick Stintzi } \\
\text { Germany }\end{array}$ & $\begin{array}{l}\text { Jihua Tang } \\
\text { China }\end{array}$ \\
\hline $\begin{array}{l}\text { Leif Skot } \\
\text { UK }\end{array}$ & $\begin{array}{l}\text { Maciej Stobiecki } \\
\text { Poland }\end{array}$ & $\begin{array}{l}\text { Georgia Tanou } \\
\text { Greece }\end{array}$ \\
\hline $\begin{array}{l}\text { Mark Smith } \\
\text { Canada }\end{array}$ & $\begin{array}{l}\text { Lucia Strader } \\
\text { USA }\end{array}$ & $\begin{array}{l}\text { Celine Tasset } \\
\text { Australia }\end{array}$ \\
\hline $\begin{array}{l}\text { Kevin Smith } \\
\text { USA }\end{array}$ & $\begin{array}{l}\text { Koichi Sugimoto } \\
\text { Japan }\end{array}$ & $\begin{array}{l}\text { Massimiliano Tattini } \\
\text { Italy }\end{array}$ \\
\hline $\begin{array}{l}\text { Petr Smykal } \\
\text { Czech Republic }\end{array}$ & $\begin{array}{l}\text { Genlou Sun } \\
\text { Canada }\end{array}$ & $\begin{array}{l}\text { Noemi Tel-Zur } \\
\text { Israel }\end{array}$ \\
\hline $\begin{array}{l}\text { David Smyth } \\
\text { Australia }\end{array}$ & $\begin{array}{l}\text { Qi Sun } \\
\text { USA }\end{array}$ & $\begin{array}{l}\text { Francisco Tenllado } \\
\text { Spain }\end{array}$ \\
\hline $\begin{array}{l}\text { Kimberley Snowden } \\
\text { New Zealand }\end{array}$ & $\begin{array}{l}\text { Wenxian Sun } \\
\text { China }\end{array}$ & $\begin{array}{l}\text { Jason Terpolilli } \\
\text { Australia }\end{array}$ \\
\hline $\begin{array}{l}\text { Michal Sochor } \\
\text { Czech Republic }\end{array}$ & $\begin{array}{l}\text { Sridevi Sureshkumar } \\
\text { Australia }\end{array}$ & $\begin{array}{l}\text { Valeria Terzi } \\
\text { Italy }\end{array}$ \\
\hline $\begin{array}{l}\text { Moon-Soo Soh } \\
\text { Korea, South }\end{array}$ & $\begin{array}{l}\text { Fedora Sutton } \\
\text { USA }\end{array}$ & $\begin{array}{l}\text { Pilar S. Testillano } \\
\text { Spain }\end{array}$ \\
\hline $\begin{array}{l}\text { Juan Solomon } \\
\text { USA }\end{array}$ & $\begin{array}{l}\text { Stephanie Swarbreck } \\
\text { UK }\end{array}$ & $\begin{array}{l}\text { Anja Thalhammer } \\
\text { Germany }\end{array}$ \\
\hline $\begin{array}{l}\text { Rentao Song } \\
\text { China }\end{array}$ & $\begin{array}{l}\text { Kelly Swarts } \\
\text { USA }\end{array}$ & $\begin{array}{l}\text { Nguyen Phuong Thao } \\
\text { Viet Nam }\end{array}$ \\
\hline $\begin{array}{l}\text { Gabriella Sonnante } \\
\text { Italy }\end{array}$ & $\begin{array}{l}\text { Laszlo Szabados } \\
\text { Hungary }\end{array}$ & $\begin{array}{l}\text { Guenter Theissen } \\
\text { Germany }\end{array}$ \\
\hline $\begin{array}{l}\text { Jose Miguel Soriano Soriano } \\
\text { Italy }\end{array}$ & $\begin{array}{l}\text { Jedrzej Szymanski } \\
\text { Israel }\end{array}$ & $\begin{array}{l}\text { Gerhard Thiel } \\
\text { Germany }\end{array}$ \\
\hline $\begin{array}{l}\text { Nathan Springer } \\
\text { USA }\end{array}$ & $\begin{array}{l}\text { Alice Tadiello } \\
\text { Italy }\end{array}$ & $\begin{array}{l}\text { Marco Thines } \\
\text { Germany }\end{array}$ \\
\hline $\begin{array}{l}\text { Sheshshayee Sreeman } \\
\text { India }\end{array}$ & $\begin{array}{l}\text { Helen Tai } \\
\text { Canada }\end{array}$ & $\begin{array}{l}\text { Sébastien Thomine } \\
\text { France }\end{array}$ \\
\hline $\begin{array}{l}\text { Nese Sreenivasulu } \\
\text { Germany }\end{array}$ & $\begin{array}{l}\text { Junji Takabayashi } \\
\text { Japan }\end{array}$ & $\begin{array}{l}\text { Michael Thomson } \\
\text { Philippines }\end{array}$ \\
\hline $\begin{array}{l}\text { Rakesh Srivastava } \\
\text { India }\end{array}$ & $\begin{array}{l}\text { Taku Takahashi } \\
\text { Japan }\end{array}$ & $\begin{array}{l}\text { Corinna Thurow } \\
\text { Germany }\end{array}$ \\
\hline $\begin{array}{l}\text { Margaret Staton } \\
\text { USA }\end{array}$ & $\begin{array}{l}\text { Toshiyuki Takai } \\
\text { Japan }\end{array}$ & $\begin{array}{l}\text { Li Tian } \\
\text { USA }\end{array}$ \\
\hline $\begin{array}{l}\text { Katherine Steele } \\
\text { UK }\end{array}$ & $\begin{array}{l}\text { Naoki Takata } \\
\text { Japan }\end{array}$ & $\begin{array}{l}\text { Denise Tieman } \\
\text { USA }\end{array}$ \\
\hline $\begin{array}{l}\text { Nils Stein } \\
\text { Germany }\end{array}$ & $\begin{array}{l}\text { Shigeo Takumi } \\
\text { Japan }\end{array}$ & $\begin{array}{l}\text { Peter Tiffin } \\
\text { USA }\end{array}$ \\
\hline $\begin{array}{l}\text { Trevor Stevenson } \\
\text { Australia }\end{array}$ & $\begin{array}{l}\text { Tsuyoshi Tanaka } \\
\text { Japan }\end{array}$ & $\begin{array}{l}\text { Mikko Tikkanen } \\
\text { Finland }\end{array}$ \\
\hline
\end{tabular}




\author{
Michael Timko \\ USA
}

Louis Tisa

USA

Alain Tissier
Germany
Takayuki Toh
Germany
Seiichi Toki
Japan
J Tokuhisa
USA

Alessandro Tondelli Italy

Kinya Toriyama

Japan

Paola Tosi

UK

Emily Tozzi

USA

Lam-Son Tran

Japan

Leena Tripathi

Kenya

Vineeta Tripathi

India

Tokuji Tsuchiya

China

Lilu Tu

China

Matthew Tucker

Australia

Paul Tudzynski

Germany

Rickie Turley

USA

Ludmila Tyler

USA

Joshua Udall

USA

Saneyoshi Ueno

Japan

Kristian Ullrich

Germany
Roman Ulm

Switzerland

Peter Ulvskov

Denmark

\section{Mikihisa Umehara}

Japan

Shimpei Uraguchi

Japan

Bjorn Usadel

Germany

Roland Valcke

Belgium

Esther Van Der Knaap

USA

C. Gerard Van Der Linden

Netherlands

Leon Van Eck

USA

Joost Van Heerwaarden

Netherlands

Sjaak Van Heusden

Netherlands

Eibertus Nicolaas Van Loo

Netherlands

Gerben Van Ooijen

UK

Leo Van Overbeek

Netherlands

Klaas Van Wijk

USA

Michiel Vandenbussche

France

Kranthi Varala

USA

Felicity Vear

France

Leonardo Velasco

Sri Lanka

Ignazio Verde

Italy

Paul Verslues

Taiwan

Filipe Victoria

Brazil
Sabina Vidal

Uruguay

Maria Lucia Carneiro Vieira

Brazil

Martin Vila Aiub

Argentina

Patrick Vincourt

France

Pavel Vítámvás

Czech Republic

Paola Vittorioso

Italy

Melane Alethea Vivier

South Africa

Konstantinos Vlachonasios

Greece

Thomas Vogt

Germany

Hugo Volkaert

Thailand

Albrecht Von Arnim

USA

Mandy Walker

Australia

John Walker

USA

Ian Wallace

USA

John Walsh

UK

Michael H. Walter

Germany

Yongfang Wan

UK

Aiming Wang

Canada

Yu Fu Wang

China

Daowen Wang

China

Li Wang

China

Lijun Wang

China 
Ming-Bo Wang

Australia

Mei Wang

China

Chung-Ju Wang

Taiwan

Junqi Wang

China

Baichen Wang

China

Guixi Wang

China

Hao Wang

Hong Kong

Jianying Wang

USA

Shucai Wang

China

Xingjun Wang

China

Xuelu Wang

China

Yi Wang

China

Ying Wang

China

Hao Wang

USA

Brian Waters

USA

Jill Wegrzyn

USA

Dongmei Wei

China

Shu Wei

China

Ralf Welsch

Germany

Susann Wicke

Germany

Olivia Wilkins

USA

Eva-Maria Willing

Germany
Iain Wilson

Australia

Karl Wilson

USA

Zoe Wilson

UK

Kamil Witek

UK

Claus-Peter Witte

Germany

Sebastian Wolf

Germany

Marnin Wolfe

USA

Anne-Marie Wolters

Netherlands

Darren Wong

Canada

Feibo Wu

China

Xiaoming Wu

China

Yisheng Wu

USA

Nelson Wulff

Brazil

Bernhard Wurzinger

Austria

Gui-Xian Xia

China

Deyu Xie

USA

Zhixin Xie

USA

Yongzhong Xing

China

Kevin Xiong

USA

Changjie Xu

China

Chunming Xu

China

Huini Xu

China
Mingliang Xu

China

Lin Xu

China

Liangjiao Xue

USA

Yukinori Yabuta

Japan

Igor Yakovlev

Norway

Toshiya Yamamoto

Japan

Jun Yan

USA

Huimin Yang

China

Ming Yang

USA

Qing Yang

China

Tae-Jin Yang

Korea, South

Yi Yang

China

Guangxiao Yang

China

Yinong Yang

USA

Zhong-Nan Yang

China

Yu Yanli

China

Quanhong Yao

China

Yingyin Yao

China

Hideshi Yasui

Japan

Huaxun Ye

USA

Zhuoliang Ye

USA

Kuo-Chen Yeh

Taiwan 


\begin{tabular}{|c|c|c|}
\hline $\begin{array}{l}\text { Bin Yi } \\
\text { China }\end{array}$ & $\begin{array}{l}\text { Deqiang Zhang } \\
\text { China }\end{array}$ & $\begin{array}{l}\text { Shao Jian Zheng } \\
\text { China }\end{array}$ \\
\hline $\begin{array}{l}\text { Yanhai Yin } \\
\text { USA }\end{array}$ & $\begin{array}{l}\text { Dong Zhang } \\
\text { USA }\end{array}$ & $\begin{array}{l}\text { Wenguang Zheng } \\
\text { USA }\end{array}$ \\
\hline $\begin{array}{l}\text { Cheol-Min Yoo } \\
\text { USA }\end{array}$ & $\begin{array}{l}\text { Jingjuan Zhang } \\
\text { Australia }\end{array}$ & $\begin{array}{l}\text { Jun Zheng } \\
\text { China }\end{array}$ \\
\hline $\begin{array}{l}\text { Mi-Jeong Yoo } \\
\text { USA }\end{array}$ & $\begin{array}{l}\text { Jian Zhang } \\
\text { Canada }\end{array}$ & $\begin{array}{l}\text { Adele Zhou } \\
\text { USA }\end{array}$ \\
\hline $\begin{array}{l}\text { Keisuke Yoshida } \\
\text { Japan }\end{array}$ & $\begin{array}{l}\text { Jinfa Zhang } \\
\text { USA }\end{array}$ & $\begin{array}{l}\text { Meixue Zhou } \\
\text { Australia }\end{array}$ \\
\hline $\begin{array}{l}\text { Kumi Yoshida } \\
\text { Japan }\end{array}$ & $\begin{array}{l}\text { Jin-Song Zhang } \\
\text { China }\end{array}$ & $\begin{array}{l}\text { Weijun Zhou } \\
\text { China }\end{array}$ \\
\hline $\begin{array}{l}\text { Keiko Yoshioka } \\
\text { Canada }\end{array}$ & $\begin{array}{l}\text { Lizhi Zhang } \\
\text { USA }\end{array}$ & $\begin{array}{l}\text { Yongming Zhou } \\
\text { China }\end{array}$ \\
\hline $\begin{array}{l}\text { Bin Yu } \\
\text { USA }\end{array}$ & $\begin{array}{l}\text { Guoquan Zhang } \\
\text { China }\end{array}$ & $\begin{array}{l}\text { Jianhua Zhu } \\
\text { USA }\end{array}$ \\
\hline $\begin{array}{l}\text { Jianming Yu } \\
\text { USA }\end{array}$ & $\begin{array}{l}\text { Liang Zhang } \\
\text { China }\end{array}$ & $\begin{array}{l}\text { Changfu Zhu } \\
\text { Spain }\end{array}$ \\
\hline $\begin{array}{l}\text { Tien-Shin Yu } \\
\text { Taiwan }\end{array}$ & $\begin{array}{l}\text { Xian-Chun Zhang } \\
\text { China }\end{array}$ & $\begin{array}{l}\text { Danmeng Zhu } \\
\text { China }\end{array}$ \\
\hline $\begin{array}{l}\text { Xiaolin Yu } \\
\text { China }\end{array}$ & $\begin{array}{l}\text { Yansheng Zhang } \\
\text { China }\end{array}$ & $\begin{array}{l}\text { Jörg Ziegler } \\
\text { Germany }\end{array}$ \\
\hline $\begin{array}{l}\text { Jingjuan Yu } \\
\text { China }\end{array}$ & $\begin{array}{l}\text { Zhanyuan Zhang } \\
\text { USA }\end{array}$ & $\begin{array}{l}\text { Agnieszka Zienkiewicz } \\
\text { USA }\end{array}$ \\
\hline $\begin{array}{l}\text { Olga Yurchenko } \\
\text { USA }\end{array}$ & $\begin{array}{l}\text { Zhiming Zhang } \\
\text { China }\end{array}$ & $\begin{array}{l}\text { Piotr Ziolkowski } \\
\text { Poland }\end{array}$ \\
\hline $\begin{array}{l}\text { Lorenzo Zacarias } \\
\text { Sri Lanka }\end{array}$ & $\begin{array}{l}\text { Huixian Zhao } \\
\text { China }\end{array}$ & $\begin{array}{l}\text { Kaijing Zuo } \\
\text { China }\end{array}$ \\
\hline $\begin{array}{l}\text { Zbynek Zdrahal } \\
\text { Czech Republic }\end{array}$ & $\begin{array}{l}\text { Meixia Zhao } \\
\text { USA }\end{array}$ & $\begin{array}{l}\text { Matias Zurbriggen } \\
\text { Germany }\end{array}$ \\
\hline $\begin{array}{l}\text { Dali Zeng } \\
\text { China }\end{array}$ & $\begin{array}{l}\text { Hongbo Zhao } \\
\text { China }\end{array}$ & $\begin{array}{l}\text { Eva Zyprian } \\
\text { Germany }\end{array}$ \\
\hline $\begin{array}{l}\text { Chunquan Zhang } \\
\text { USA }\end{array}$ & $\begin{array}{l}\text { Chengchao Zheng } \\
\text { China }\end{array}$ & \\
\hline
\end{tabular}

